\title{
The struggles of cancer centers
}

$\mathrm{n}$ past editorials, I said that I would revisit the topic about the War on Cancer. I have been reflecting a lot on how things have progressed since the Cancer Act was passed in 1971. Cancer centers were a major theme of the Cancer Act, and before this Act was passed we had three freestanding cancer centers in the US. Now we have 63-the vast majority of which are referred to as 'matrix centers', which means they are integrated within the structure of university medical schools and hospitals. The matrix set-up helped us establish and run new cancer centers across the country much faster than independent centers, and at less cost than building new, freestanding ones.

The downside, however, of these matrix centers is that historically they have often run afoul of the departmental structure of medical schools. Such centers are often centers in name only. More energy is often expended in turf struggles between the departments of medicine and cancer centers over the operation of the sections of Medical Oncology than over innovations in cancer care. This is a recurring theme that has dogged National Cancer Institute (NCI) Cancer Centers since their inception in 1972.

Was there a better model? Possibly. The Columbia College of Physicians and Surgeons (CP\&S) established the first university-based freestanding cancer hospital. However, even this center was dogged by some of the problems that affect the matrix model. In November 2007, I interviewed Dr Alfred Gellhorn, the first Director of the Francis Delafield Cancer Hospital.

Gellhorn was trained as a general surgeon but retrained in Internal Medicine. In 2007, he was involved in his fourth successful career since leaving the Delafield Cancer Hospital. I asked Dr Gellhorn to explain how the Delafield Cancer Hospital was established. "It started in 1952. A CP\&S breast surgeon by the name of Christian Hagenson persuaded the city to build a cancer hospital. Hagenson could never find anybody in Internal Medicine who was interested in cancer because the Chairman of Medicine, Robert Loeb, felt that this was a disease that a good internist would have nothing to do with, because you couldn't cure it. I had absolutely unbounded admiration for Bob Loeb. He was a magnificent clinician.... and a wonderful teacher. I think he just had a blind spot when it came to cancer. I think that the reason that Medical Oncology got such a slow start had to do with the attitude of people in Internal Medicine."

Gellhorn explained that Loeb would not consider rotating CP\&S house staff through a cancer hospital, but said they were eventually able to get approval for their own residency program. "I was able to recruit some wonderful colleagues, such as Paul Marks, Elliot Osserman, John Ultmann, Bernie Weinstein, Helen Ranney, Franco Muggia, among others. My first chief resident was Jim Holland." At the time, Gellhorn was the Director of the Institute of Cancer Research. The Delafield was its clinical arm that also had laboratories. "Loeb tolerated me but he was fond of saying rather openly, "Alfred, you're a part of the lunatic fringe." Loeb then retired and an even more pessimistic and indifferent clinician came in as Chair of Medicine, Stanley Bradley. Stan Bradley sent two guys to replace me in 1968 who had no connection to cancer and it was closed shortly thereafter."

Despite the obvious need for, and the success of the Delafield (his trainees are a who's who of cancer medicine), two Chairs of Medicine closed it. Their actions set the template for most NCI matrix centers today. Delafield was a good model, just not freestanding enough. CP\&S received its NCI designation in 1974 . Since then, like a lot of matrix centers, they have struggled to find their Cancer Center identity. I am sure, for those who knew of the Delafield, there have been many times when they wished it was still open.

Dr Gellhorn died peacefully in March 2008 at the age of 94 . He was a great mentor and a pioneer in the cancer field. Not many people, having been told they were part of the "lunatic fringe" by one of the greats in medicine, would have had the fortitude to continue. He did. For that, and all the many luminaries he trained, we all owe him a debt of gratitude. Thanks Alfred.

doi:10.1038/nrclinonc.2009.51
Vincent T DeVita Jr is the Editor-in-Chief of Nature Reviews Clinical Oncology.

Competing interests The author declared no competing interests. 